\title{
Use of the Rigid Band Formalism to Interpret the Relationship between $O$ Chemical Potential and Electron Concentration in $\mathrm{La}_{1-x} \mathrm{Sr}_{x} \mathrm{CoO}_{3-\delta}$
}

\author{
Martijn H. R. Lankhorst, H. J. M. Bouwmeester, and H. Verweij \\ University of Twente, Department of Chemical Technology, Laboratory of Inorganic Materials Science, \\ P.O. Box 217, 7500 AE Enschede, The Netherlands
}

(Received 18 March 1996; revised manuscript received 25 June 1996)

\begin{abstract}
The equilibrium oxygen chemical potential of mixed oxygen ion and electron conducting perovskites $\mathrm{La}_{1-x} \mathrm{Sr}_{x} \mathrm{CoO}_{3-\delta}$ was measured as a function of $\delta$ and $x$ by high temperature oxygen coulometric titration. An almost linear decrease in the chemical potential is observed with increasing net electron concentration. The observed behavior is interpreted to reflect the corresponding change in the Fermi level upon gradually filling up states in a broad electron band with electrons induced by vacancy formation and Sr doping. Assuming a rigid band formalism, an apparent density of states at the Fermi level was calculated, the value of which is in good agreement with that obtained from XPS. [S0031-9007(96)01314-2]

PACS numbers: 71.20.Eh, 61.72.Ji, 66.30.Fq
\end{abstract}

Many of the $3 d$ transition metal perovskite-type oxides are characterized by increased lattice oxygen vacancy formation at high temperatures, which results in substantial departure from oxygen stoichiometry. Vacancy formation is accompanied by a proportional change in the average valency of either the transition metal or oxygen ions. The relatively high vacancy mobility in conjunction with the metalliclike electronic conductivity causes these materials to be permeable for oxygen gas [1], which is of interest for various applications. In addition, many of the $3 d$ transition metal perovskites are studied for special electronic and magnetic transitions which may occur either as a function of temperature or composition [2-4]. In the ZSA theory [5], the electronic structure of these oxides is interpreted in terms of the competition between charge disproportionation of the Mott Hubbart type, $d_{i}^{n} d_{j}^{n} \leftrightarrows d_{i}^{n-1} d_{j}^{n+1}$, and charge transfer $d_{i}^{n} \leftrightarrows d_{i}^{n+1} \underline{L}$, where $d^{n}$ denotes the $n$-electron $3 d$ state of the transition metal and $\underline{L}$ denotes a hole in the wide anion valence band. Although charge disproportionation was not observed in $\mathrm{LaCoO}_{3}$ by XAS [3], Sarma et al. [4] state that for this material the influence of both types of charge fluctuation is of the same order of magnitude, leading to a very mixed character of the ground state. Charge disproportionation was used to model oxygen nonstoichiometry [6] and Seebeck data [7] of $\mathrm{La}_{1-x} \mathrm{Sr}_{x} \mathrm{CoO}_{3-\delta}$. In both cases, however, the best fit implied the simultaneous presence of unrealistically high concentrations of $\mathrm{Co}^{2+}$ and $\mathrm{Co}^{4+}$. In Sr-doped $\mathrm{LaCoO}_{3}$, significant spectral intensities at the Fermi energy have been observed by Sarma et al. [4] using UPS and bremsstrahlung isochromat spectroscopy. They concluded that electron hole states, created by substitution of $\mathrm{La}^{3+}$ by $\mathrm{Sr}^{2+}$, overlap the top of the wide oxygen $2 p$ band giving rise to a mixed-valency metallic compound. Furthermore, conductivity and Seebeck measurements [7] at high temperatures also indicate metalliclike properties for Sr-doped $\mathrm{LaCoO}_{3}$. These observations favor the interpretation in terms of itinerant electron behavior in these solids.
The aim of this study is, on the one hand, to improve existing oxygen nonstoichiometry models for the compounds $\mathrm{La}_{1-x} \mathrm{Sr}_{x} \mathrm{CoO}_{3-\delta}$ by incorporating the delocalized nature of the conduction electrons. One the other hand, it is aimed at unraveling details of the electronic structure of these oxides by making use of the fact that the number of conduction electrons is coupled to the number of oxygen vacancies. Hence, the conduction electron concentration can be controlled in situ by adjusting the oxygen vacancy concentration using, for instance, an oxygen coulometric titration cell. With such cell, thermodynamic properties of oxygen vacancies and electrons in the oxide can be studied by analyzing the oxygen partial pressure and temperature dependence of the oxygen vacancy concentration. It is shown that in situ adjustment of the vacancy concentration has exactly the same effect on the oxygen chemical potential as changing the $\mathrm{Sr}^{2+}$-dopant level. The results are in agreement with the assumption of a rigid band model (RBM) to be discussed later.

In a high temperature coulometric titration experiment the oxide sample is enclosed in a sealed electrochemical cell. The oxygen stoichiometry of the sample is adjusted by electrochemically pumping oxygen into or out of the cell using an oxide solid electrolyte. The oxygen partial pressure inside the sealed compartment is determined from the $E M F$ across another oxide solid electrolyte using identical metallic probes.

The oxygen pumped into the cell is incorporated into the oxide lattice as two regular lattice oxygen ions $\left(\mathrm{O}_{\mathrm{O}}^{x}\right)$, at the expense of two lattice oxygen vacancies $\left(V_{\mathrm{O}}^{\prime \prime}\right)$ and four electrons $\left(e^{\prime}\right)$,

$$
\mathrm{O}_{2}+2 V_{\mathrm{O}}^{\cdot}+4 e^{\prime} \leftrightarrows 2 \mathrm{O}_{\mathrm{O}}^{x}
$$

where the Kröger-Vink notation [8] is used. The equilibrium condition for reaction (1) reads

$$
\mu_{\mathrm{O}_{2}}=-2 \mu_{V_{\mathrm{o}}^{\prime \prime}}-4 \mu_{e},
$$

where $\mu_{i}$ denotes the chemical potential of species $i$. Since vacancies and lattice oxygens are bounded by the 
requirement of a constant number of sites $3 N, \mu_{V_{0}}$ and $\mu_{\mathrm{O}_{\mathrm{O}}^{x}}$ cannot be defined independent from each other. In Eq. (2), $\mu_{\mathrm{O}_{0}^{x}}$ can be neglected when $\mu_{V_{0}^{*}}$ is regarded as the chemical potential of a vacancy building unit.

In traditional point defect models [9], chemical potentials are derived based on the assumption that point defects can be treated as noninteracting and ideally diluted species. The resulting chemical potentials consist of a constant Helmholtz free energy of formation and a configurational entropy which originates from the random distribution of all defects over the available lattice sites. In the case of oxygen vacancy building units, this formalism leads to the following expression for $\mu_{V_{0}}$ :

$$
\mu_{V_{0}^{*}}=\mu_{V_{0}^{*}}^{0}-T\left\{k_{B} \ln [(3-\delta) / \delta]\right\},
$$

where $\delta$ is the oxygen nonstoichiometry parameter and $\mu_{V_{0}^{\prime}}^{0}$ contains the energy of formation of oxygen vacancy building units in addition to a constant energy and entropy originating from phonon contributions.

It is expected that an expression for $\mu_{e}$ similar to Eq. (3) holds only when the electrons are localized near the lattice sites, occupy an extremely narrow band, or occupy nearly empty or filled bands. In the case of a partially filled broad electron band the electron chemical potential or Fermi level is approximately equal to the energy of the highest occupied electron state. Electron states within a few $k_{B} T$ of the Fermi level are partially filled, but this effect can be neglected when the bandwidth is large compared with $k_{B} T$. The overall electron entropy thus has negligible influence on the electron chemical potential. In order to predict changes in the Fermi level with compositional variations, it is often assumed that the bands are rigid when atoms are added or removed from the solid. In this model, the Fermi level moves upwards as electrons are donated to the band, at a rate determined by the density of states at the Fermi level. Although in this simple model, commonly referred as electron gas RBM [10], important features like screening, electron correlation, and exchange are neglected, observed chemical potential variations for alkali metal intercalated transition metal dichalcogenides have been found to correlate well with density of states calculations [11] and x-ray absorption measurements [12]. It was even suggested to use the electronic structure of some intercalates as a criterion for the applicability as electrode material [13].

McKinnon and Selwyn [14] criticized the validity of the electron gas RBM. With reference to the screened impurity RBM developed by Friedel [15], these authors propose that, when impurities are added or removed, the entire band shifts due to the perturbation constituted by the screened Coulomb potential of the ionized impurity. Since the latter shift is exactly opposite to the shift in the Fermi level expected from the change in electron occupancy, the resulting value of the Fermi level is unaffected. Sellmyer [10] concluded that both models would have the same implications for an experiment in which energies are measured only relative to the bottom of the band. However, in this study energies are measured on an absolute scale, and therefore the results depend on which model is applicable.

Measurements were performed using the electrochemical cell shown in Fig. 1. A cylindrical disk of $\mathrm{La}_{1-x} \mathrm{Sr}_{x} \mathrm{CoO}_{3-\delta}$ was placed in a small cylindrical $\mathrm{ZY} 13\left(\mathrm{Zr}_{0.87} \mathrm{Y}_{0.13} \mathrm{O}_{1.935}\right)$ box, which was sealed to a ZY13 disk by a Pyrex glass ring. Pt-based electrodes were painted on both sides of the disk and the box. The inner electrodes were connected to a thin Pt tape, which was led to the outside via the Pyrex glass ring. In the cell arrangement shown in Fig. 1, the EMF over the ZY13 disk is used to determine the oxygen partial pressure in the cell $P_{\mathrm{O}_{2}}^{\text {cell }}$. The $E M F$ is related to the difference in oxygen chemical potential over the ZY13 disk $\Delta \mu_{\mathrm{O}_{2}}$ as given by

$$
E M F=\Delta \mu_{\mathrm{O}_{2}} / 4 e=\left(k_{B} T / 4 e\right) \ln \left(P_{\mathrm{O}_{2}}^{\text {ref }} / P_{\mathrm{O}_{2}}^{\text {cell }}\right),
$$

where $k_{B}$ is Boltzmann's constant, $T$ the temperature, $e$ the elementary charge, and $P_{\mathrm{O}_{2}}^{\mathrm{ref}}$ the oxygen partial pressure of the reference gas. The ZY13 box is used for electrochemical pumping of oxygen into or out of the cell. The current measured is related to the oxygen pumping rate in accordance with Faraday's law. Further details are discussed elsewhere [16].

The value of $\mu_{\mathrm{O}_{2}}$ is calculated by substituting the measured value of $P_{\mathrm{O}_{2}}^{\text {cell }}$ into the expression for the gasphase oxygen chemical potential taken from Ref. [17]. The entropy part $s_{\mathrm{O}_{2}}$ of $\mu_{\mathrm{O}_{2}}$, defined by

$$
s_{\mathrm{O}_{2}}=-\left(\partial \mu_{\mathrm{O}_{2}} / \partial T\right)_{\delta},
$$

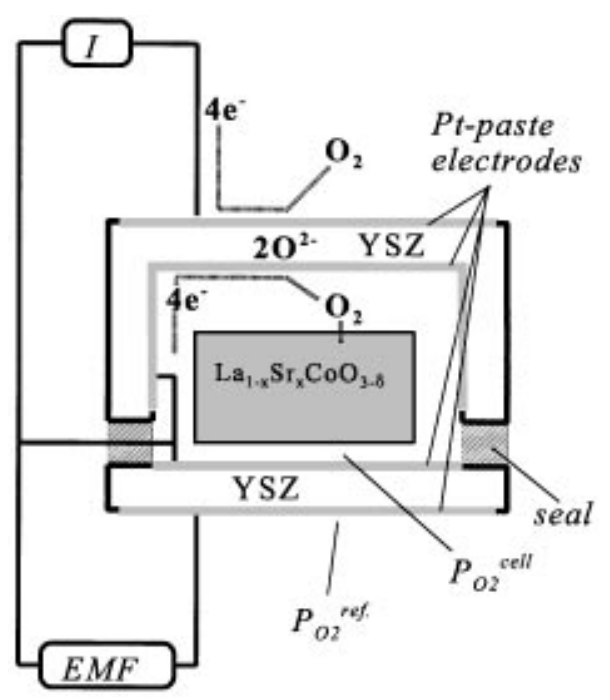

FIG. 1. Electrochemical cell used for oxygen titration experiments on $\mathrm{La}_{1-x} \mathrm{Sr}_{x} \mathrm{CoO}_{3-\delta}$. Two ZY13 solid electrolytes are used. One is used to measure the $P_{\mathrm{O}_{2}}$ in the cell [cf. Eq. (4)] and the other to pump oxygen into or out of the cell. 
is obtained by monitoring the open cell $E M F$ as a function of temperature at constant oxygen vacancy concentration. The energy part $\epsilon_{\mathrm{O}_{2}}$ is calculated from $\mu_{\mathrm{O}_{2}}=$ $\epsilon_{\mathrm{O}_{2}}-T s_{\mathrm{O}_{2}}$. Both parameters are directly related to the respective energy and entropy of lattice oxygen vacancies and conduction electrons. Between the controlled temperature variations, the oxygen stoichiometry of the sample is adjusted to a specific value by electrochemical titration. In this study, these measurements were performed on three $\mathrm{La}_{1-x} \mathrm{Sr}_{x} \mathrm{CoO}_{3-\delta}$ samples with Sr-dopant levels $x=0.2,0.4$, and 0.7 .

The measured entropy of oxygen incorporation into $\mathrm{La}_{0.8} \mathrm{Sr}_{0.2} \mathrm{CoO}_{3-\delta}$ is shown in Fig. 2. Its value increases with increasing oxygen vacancy concentration. Neglecting the entropy of the conduction electrons, Eq. (3) leads to the following expression relating $s_{\mathrm{O}_{2}}$ to $\delta$

$$
s_{\mathrm{O}_{2}}=s^{0}+2 k_{B} \ln [\delta /(3-\delta)],
$$

where $s^{0}$ is a constant. Note that $s_{\mathrm{O}_{2}}$ is independent of temperature. Figure 2 shows that experimental data for $s_{\mathrm{O}_{2}}$, evaluated by taking the partial derivative of the $\mu_{\mathrm{O}_{2}}-T$ curve, is in perfect agreement with the theoretical data calculated using Eq. (6) and $s^{0}=6.85 \times$ $10^{-4} \mathrm{eV} / \mathrm{K}$. The good agreement noted with the expression of the configurational entropy of oxygen vacancy building units supports the correctness of the assumptions of randomly distributed oxygen vacancies and that of a negligibly small contribution of the entropy of conduction electrons. From Fig. 2, it is further seen that the measured energy of oxygen incorporation into $\mathrm{La}_{0.8} \mathrm{Sr}_{0.2} \mathrm{CoO}_{3-\delta}$ decreases almost linearly with increasing nonstoichiometry, but is nearly temperature independent. In principle, such a decrease in $\epsilon_{\mathrm{O}_{2}}$ can be explained either in terms of repulsive coulombic vacancy interactions or in terms of the increase in Fermi level due to filling of the band (electron gas $R B M$ ). However, the first explanation is less likely since no decrease in $\epsilon_{\mathrm{O}_{2}}$ with increasing $\delta$ has been ob-

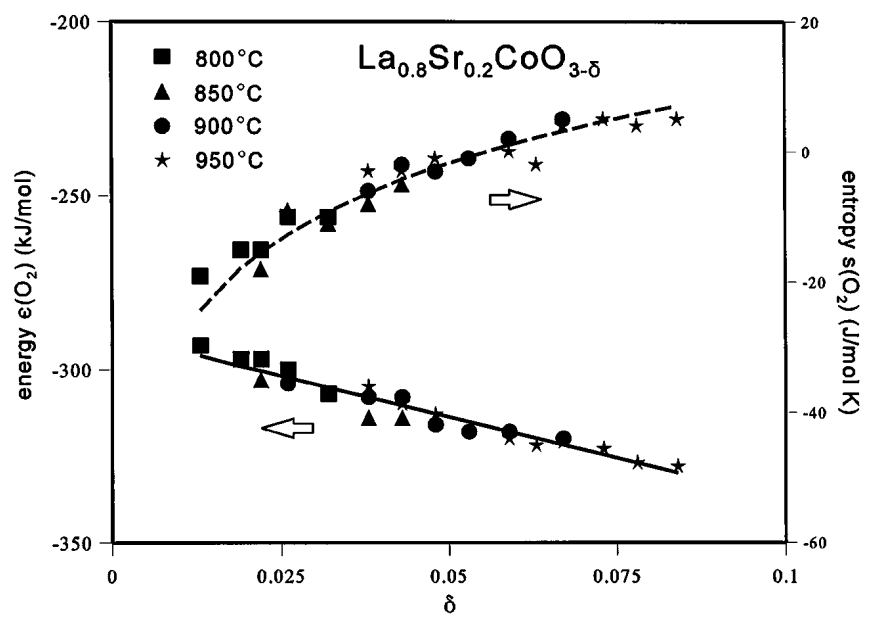

FIG. 2. Plot of the partial energy $\left(\epsilon_{\mathrm{O}_{2}}\right)$ and entropy $\left(s_{\mathrm{O}_{2}}\right)$ of $\mathrm{La}_{0.8} \mathrm{Sr}_{0.2} \mathrm{CoO}_{3-\delta}$ as a function of oxygen nonstoichiometry, $\delta$. The dashed line indicates the fit by Eq. (6). served in the related structures $\mathrm{La}_{1-x} \mathrm{Sr}_{x} \mathrm{FeO}_{3}$ [18]. In these oxides, the values of the electric conduction [19] are much smaller than in $\mathrm{La}_{1-x} \mathrm{Sr}_{x} \mathrm{CoO}_{3}$ [7], and hence screening of at least the coulombic part of vacancy interactions in the cobalt-containing oxides is expected to be more effective. The analysis of data for $x=0.4$ and 0.7 , which compounds exhibit high vacancy concentration, showed that additional ionic contributions arise in both $\epsilon_{\mathrm{O}_{2}}$ and $s_{\mathrm{O}_{2}}$. Both contributions compensate each other in the overall value of $\mu_{\mathrm{O}_{2}}$. A detailed presentation of experimental data from this study will be presented in a forthcoming paper.

In Fig. 3, the equilibrium oxygen chemical potential determined for compounds with different $\mathrm{Sr}$ contents is plotted as a function of oxygen nonstoichiometry. Data were obtained in the temperature range $688-913{ }^{\circ} \mathrm{C}$. Even though this is not indicated in Fig. 3, only for $\mathrm{La}_{0.3} \mathrm{Sr}_{0.7} \mathrm{CoO}_{3-\delta}$ a relatively small temperature influence on the oxygen chemical potential was recognized. For all compounds, $\mu_{\mathrm{O}_{2}}$ decreases almost linearly with increasing $\delta$. The slope of these lines is independent of $\mathrm{Sr}$ content, noting that the change in slope observed for $x=0.2$ at low vacancy concentration stems from the configurational entropy of oxygen vacancies. Figure 4 contains the same data points as presented in Fig. 3, but now $\mu_{\mathrm{O}_{2}}$ is plotted as a function of the electron occupancy in the band calculated from $2 \delta-x$. Strikingly, all data points fall onto a single line. This simple relationship between $\mu_{\mathrm{O}_{2}}$ and the electron occupancy is to a first order of approximation indpendent of temperature and composition.

Since the average valency of the $\mathrm{La} / \mathrm{Sr}$ lattice increases with decreasing $\mathrm{Sr}$ content, the observed decrease in $\mu_{\mathrm{O}_{2}}$ with decreasing $x$ at a fixed $\delta$ would suggest that oxygen ions are more strongly bound in a lattice with less Sr. The observed decrease in $\mu_{\mathrm{O}_{2}}$ with increasing $\delta$ at a fixed $x$ might be interpreted in terms of vacancy interactions. These inter-

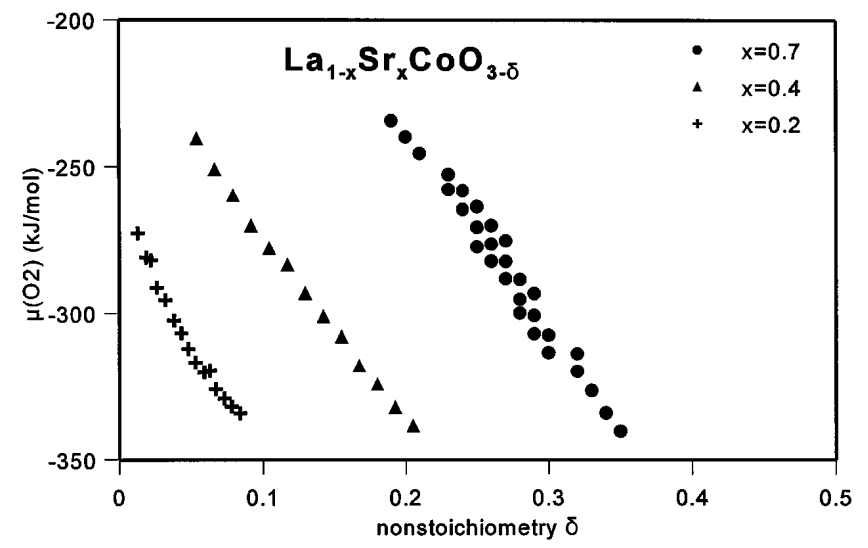

FIG. 3. Plot of $\mu_{\mathrm{O}_{2}}$ as a function of $\delta$ measured for three $\mathrm{La}_{1-x} \mathrm{Sr}_{x} \mathrm{CoO}_{3-\delta}$ samples with $x=0.2,0.4$, and 0.7. Data points were obtained in the temperature range $688-913{ }^{\circ} \mathrm{C}$. 


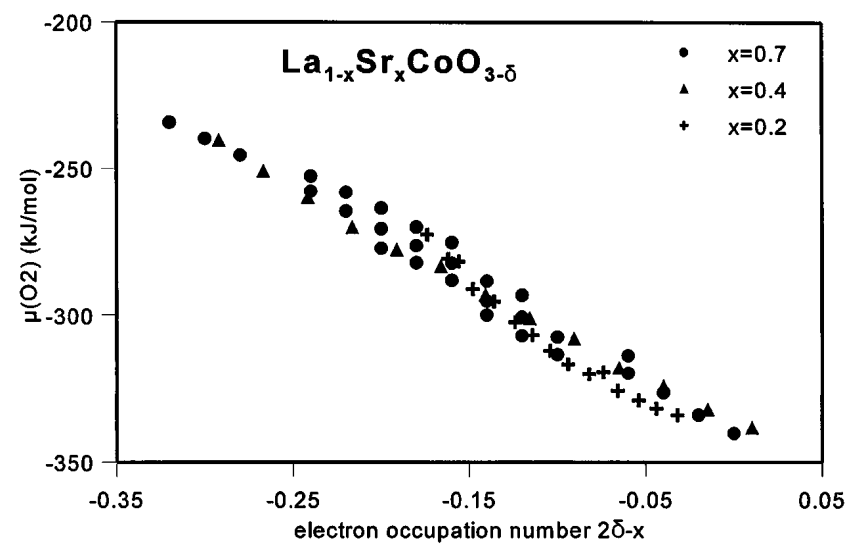

FIG. 4. Plot of $\mu_{\mathrm{O}_{2}}$ as a function of electron occupation number $2 \delta-x$ measured for three $\mathrm{La}_{1-x} \mathrm{Sr}_{x} \mathrm{CoO}_{3-\delta}$ samples with $x=0.2,0.4$, and 0.7. Data points were obtained in the temperature range $688-913{ }^{\circ} \mathrm{C}$.

pretations are based totally on ionic interactions but cannot explain the data in Fig. 4, unless it is believed that the variations in $\mu_{\mathrm{O}_{2}}$ with $x$ and with $\delta$ cancel out. A more convincing explanation that does not rely on such a coincidence is that the observed behavior in Fig. 4 results from the upward shift in Fermi level with increasing the number of electrons in the band. The latter interpretation requires that the electron band must be rigid in the sense that it does not move as a function of changes in the composition. Assuming the simple electron gas $R B M$ to hold the data of Fig. 4 enable calculation of the density of states at the Fermi level equal to $1.1 \mathrm{eV}^{-1}$ pro unit cell. The latter value is found to be in good agreement with the values of 1.0 and $1.3 \mathrm{eV}^{-1}$, which were derived from the shift in the $\mathrm{O}-2 p$ band towards the Fermi level with increasing $\mathrm{Sr}$ content as measured using XPS [4,20]. Hence, it must be concluded that in compounds $\mathrm{La}_{1-x} \mathrm{Sr}_{x} \mathrm{CoO}_{3-\delta}$ the downwards shift of the electron band as a result of screening expected in the screened impurity $R B M$ must be small compared with the increase in Fermi level with increasing electron occupancy. It should be noted that for another material the influence of screening could be more important and a similar correspondence between the density of states obtained from spectroscopic and electrochemical measurements may not be found.

To summarize, no influence of the entropy change of electrons can be identified from the data of entropy associated with oxygen incorporation into $\mathrm{La}_{0.8} \mathrm{Sr}_{0.2} \mathrm{CoO}_{3-\delta}$. The equilibrium oxygen chemical potential of $\mathrm{La}_{1-x} \mathrm{Sr}_{x} \mathrm{CoO}_{3-\delta}$ decreases almost linearly with the increasing electron occupation number. These results can be explained by the electron gas rigid band model in which electrons created during vacancy formation or decreasing the $\mathrm{Sr}$ content are assumed to be placed in a band with a relatively low density of states at the Fermi level. The shift in the electron band as a result of screening the ionized defects seems to be small.

[1] Y. Teraoka, H. M. Zhang, S. Furukawa, and N. Yamazoe, Chem. Lett. 1985, 1743 (1985).

[2] H. Taguchi, M. Shimada, and M. Koizumi, J. Solid State Chem. 29, 221-225 (1979).

[3] M. Abbate, J.C. Fuggle, A. Fujimori, A. Fujimori, L.H. Tjeng, C.T. Chen, R. Potze, G. A. Sawatzky, H. Eisaki, and S. Uchida, Phys. Rev. B 47, 16124 (1993).

[4] D. D. Sarma and A. J. Chainani, J. Solid State Chem. 111, 208 (1994).

[5] J. Zaanen, G. A. Sawatzky, and J. W. Allen, Phys. Rev. Lett. 55, 418 (1985).

[6] B. A. van Hassel, T. Kawada, N. Sakai, H. Yokokawa, M. Dokiya, and H. J. M. Bouwmeester, Solid State Ionics 66, 295 (1993).

[7] L. W. Tai, M. M. Nasrallah, H. U. Anderson, D. M. Sparlin, and S. R. Sehlin, Solid State Ionics 76, 273 (1995).

[8] F. A. Kröger and H. J. Vink, in Solid State Physics, edited by F. Seitz and D. Turnball (Academic Press, New York, 1956), Vol. 3, p. 307.

[9] F.A. Kröger, in The Chemistry of Imperfect Crystals (North-Holland, Amsterdam, 1964), Chap. 9.

[10] D. J. Sellmyer, in Solid State Physics, edited by H. Ehrenreich, F. Seitz, and D. Turnball (Academic Press, New York, 1978), Vol. 33, p. 83.

[11] J. Rouxel, in The Electronic Transfer and the Formation of Cationic Intercalation Compounds, edited by G. Nazri, R. F. Huggins, and D. F. Shriver, MRS Symposia Proceedings No. 135 (Materials Research Society, Pittsburgh, 1989), p. 431 (1989).

[12] J. R. Dahn, J. N. Reimers, T. Tiedje, Y. Gao, A. K. Sleigh, W. R. McKinnon, and S. Cramm, Phys. Rev. Lett. 68, 835 (1992).

[13] J. Molenda, Solid State Ionics 21, 263 (1986).

[14] W. R. McKinnon and L. S. Selwyn, Phys. Rev. B 35, 7275 (1987).

[15] J. Friedel, Adv. Phys. 3, 446 (1954).

[16] M. H. R. Lankhorst and H. J. M. Bouwmeester, "Determination of Oxygen Nonstoichiometry and Diffusivity in Mixed Conducting Oxides by Oxygen Coulometric Titration, Part I: Chemical Diffusion in $\mathrm{La}_{0.8} \mathrm{Sr}_{0.2} \mathrm{CoO}_{3-\delta}$ " (to be published).

[17] Commission on Thermodynamics, International Union of Pure and Applied Chemistry, International Thermodynamic Tables of the Fluid State-9, Oxygen (Blackwell Scientific Publications, Oxford, 1987).

[18] J. Mizusaki, M. Yoshihiro, S. Yamauchi, and K. Fueki, J. Solid State Chem. 67, 1 (1987).

[19] J. Mizusaki, T. Sasamoto, W. R. Cannon, and H. K. Bowen, J. Am. Ceram. Soc. 66, 247 (1983).

[20] J. P. Kemp, D. J. Beal, and P. A. Cox, J. Solid State Chem. 86, 50 (1990). 